\title{
Learning with Sparse and Biased Feedback for Personal Search
}

\author{
Michael Bendersky, Xuanhui Wang, Marc Najork and Donald Metzler \\ Google, Inc. \\ \{bemike, xuanhui, najork, mezler\}@google.com
}

\begin{abstract}
Personal search, including email, on-device, and personal media search, has recently attracted a considerable attention from the information retrieval community. In this paper, we provide an overview of challenges and opportunities of learning with implicit user feedback (e.g., click data) in personal search. Implicit user feedback provides a convenient source of supervision for ranking models in personal search. This feedback, however, has two major drawbacks: it is highly sparse and biased due to the personal nature of queries and documents. We demonstrate how these drawbacks can be overcome, and empirically demonstrate the benefits of learning with implicit feedback in the context of a large-scale email search engine ${ }^{1}$.
\end{abstract}

\section{Introduction}

Researchers have been exploring how to successfully leverage user feedback to improve search quality for over a decade [Joachims, 2002; Joachims et al., 2005]. User feedback most often comes in the form of clicks on links to search results, but may be derived from other sources, including page visits [Richardson et al., 2006], cursor tracking [Guo and Agichtein, 2012], or touch gestures [Guo et al., 2013]. Such user interaction data has been shown to be particularly useful for training learning to rank models [Agichtein et al., 2006; Richardson et al., 2006] and click-through rate prediction [Richardson et al., 2007].

However, even though the use of interactions for improving search over public search corpora (e.g., web search) is commonplace, there is little to no research regarding its use for search over personal corpora, a.k.a. personal search. Personal search has many real-life applications including (but not limited to) email search [Carmel et al., 2015; Wang et al., 2016], desktop search [Dumais et al., 2003],

\footnotetext{
${ }^{1}$ This paper is an abridged version of the paper "Learning from User Interactions in Personal Search via Attribute Parameterization" that was nominated for the Best Paper Award at WSDM 2017 conference. It also contains some material from the paper "Position Bias Estimation for Unbiased Learning to Rank in Personal Search" which appeared at WSDM 2018 conference.
}

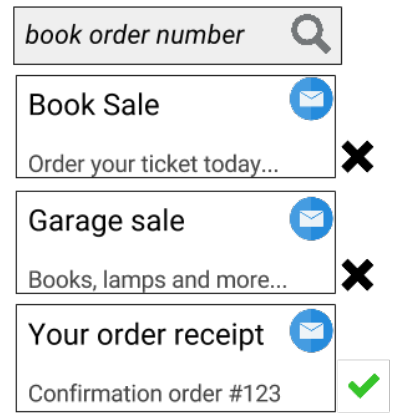

Figure 1: Illustrative example of email search results for query [book order number]. The first two results are skipped, and the last one is clicked.

and, most recently, on-device search [Kamvar et al., 2009] and personal media search [Anguera et al., 2008; Guy et al., 2018].

In all of these personal search applications, leveraging user feedback for improving search quality has been limited by its sparseness. This sparseness arises from the fact that in the personal search scenario each user has access only to their own private corpus (e.g., emails, documents or multimedia files). This means that cross-user interactions with the same item, which are common in web search (i.e., millions of users visiting the same web page) are non-existent in personal search.

Second, user queries in personal search may not generalize as well as in web search due to the private nature of the underlying corpora. For instance, one common use case in email search is retrieving some personal information of a correspondent, e.g. [marta schedule], or [from:john highest-priority] [Carmel et al., 2015]. This is very different from web search, where the most common queries are issued by multiple users with the same underlying target page in mind.

For instance, consider the email search example in Figure 1. In this case the user skipped the first two results (even though they might have more terms in common with the query book order number) and clicked on the last result. It would be impossible to directly leverage this specific interaction to learn a model for other users given the private nature of the interaction (since no other user received an email with the exact same order number). 


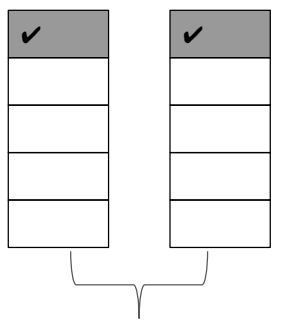

Q1

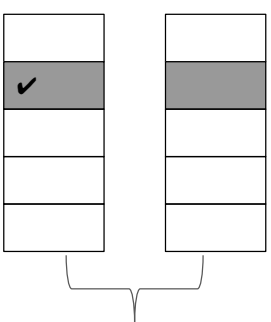

Q2
Figure 2: An illustration of selection bias in click data. The shaded documents are the relevant ones. A check mark indicates a click.

However, by aggregating non-private query and document attributes (i.e., those that exclude any personal information such as order number) across a large number of user interactions, it is possible to identify privacy-preserving querydocument associations that can be leveraged to improve search quality across all users. For instance, by using term associations, we can learn that emails with the frequent term receipt in the subject are likely to be relevant to queries containing the frequent $\mathrm{n}$-gram order number. As another example, using structural associations [Ailon et al., 2013], we can learn that emails from an online bookstore AliceBookseller.com that correspond to a subject template Your order receipt $*$ are more likely to be relevant to queries containing the frequent n-gram book order.

In addition to the sparsity problem, implicit user feedback such as click data is biased [Joachims et al., 2005; Wang et al., 2016]. For instance, consider Figure 2, which shows two queries $Q 1$ and $Q 2$. The relevant document for $Q 1$ is at position 1 and is clicked every time the query is issued. On the contrary, the relevant document for $Q 2$ is at position 2 and is clicked only half of the time, due to the user propensity to pay less attention to the lower rank results.

The problem illustrated in this example is confirmed by eye tracking studies as well, which found that the users are less likely to see, and hence click on, lower-ranked documents [Joachims et al., 2005; Richardson et al., 2007]. This click position bias leads, in turn, to selection bias - queries with clicks on lower rank positions tend to be under-represented in training data for learning to rank models, as shown by [Wang et al., 2016].

While in a public search setting such as web search selection bias could be corrected by collecting explicit human ratings, in the personal search setting collecting such ratings is much harder since raters can only label their own queries and the corresponding results. In addition, such ratings will be heavily dependent on the selected raters, creating yet another source of bias. Finally, such ratings are costly to maintain due to the dynamic nature of personal search collections. Therefore, in personal search, researchers and practitioners [Tromba et al., 2017] often have to rely on click data to optimize and evaluate ranking models.

In the next section, we provide an exposition of our recent work on dealing with the challenges of sparse and biased user feedback in the personal search setting. Then, in Section 3, we provide a brief overview of empirical evaluation of our

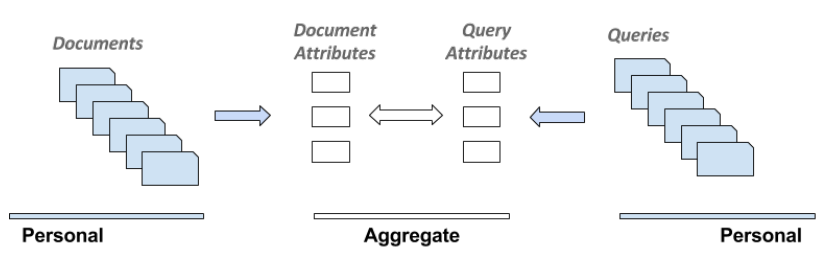

Figure 3: Attribute aggregation and matching.

methods in the context of a large-scale email search engine. We conclude the paper in Section 4.

\section{Model Overview}

In this section, we overview several models that deal with the sparsity and bias that are inherent to learning with click data in personal search, and in particular in an email search setting. We start with a brief overview of the learning to rank [Liu, 2009] methodology for email search in Section 2.1. In Section 2.2, we propose a novel way to reduce sparcity in user feedback via cross-user aggregation [Bendersky et al., 2017]. In Section 2.3 we describe how we deal with position bias in user feedback [Wang et al., 2016; Wang et al., 2018]

\subsection{Learning to Rank in Email Search}

In the most general setting, a training data for learning the optimal search ranking (a.k.a. learning to rank [Liu, 2009]) consists of a query set $Q$, where for each query $q$, we are given a list of corresponding documents $D_{q}$. Each querydocument pair ( $q \in Q, d \in D_{q}$ ), is associated with a feature vector $\mathbf{x}_{q, d}$ and a corresponding relevance label $l_{q, d}$. While in scenarios like web search, relevance labels $l_{q, d}$ are often obtained using explicit relevance judgments, in the personal search setting such as email search labels are usually derived from click data, and thus are sparse and biased.

The goal of learning to rank algorithms is to produce an optimal ranking function $s c\left(\mathbf{x}_{q, d}\right)$. There are many approaches to this problem, roughly categorized as pointwise, pairwise and listwise [Liu, 2009]. Their overview is out of the scope of this paper, however it is important to note that the techniques discussed in the next sections are agnostic to the choice of any particular approach.

Feature vector $\mathbf{x}_{q, d}$ may contain any signals derived from query $q$, document $d$ or both. In particular, for email search, the features may be derived from the message metadata, sender, recipient and textual similarity between the message and the query (see [Carmel et al., 2015] for an overview). In the next section, we demonstrate how click-based features can also be incorporated in the feature vector.

\subsection{Learning with Sparse Feedback}

Historical user click data, as observed in the search logs, can provide a powerful signal for click-through rate prediction and learning to rank models, since it directly reflects user behavior. For instance, if we observe previous interactions for a given query-document pair $(q, d)$, we may use it as a query-dependent matching feature in a feature vector $\mathbf{x}_{q, d}$ in a learning to rank model (e.g., aggregate number of 
Proceedings of the Twenty-Seventh International Joint Conference on Artificial Intelligence (IJCAI-18)

\begin{tabular}{|l|l|l|}
\hline & Document & Query \\
\hline Categories & $\begin{array}{l}\text { Small set of commonly used email labels, e.g., Purchases, } \\
\text { Promos, Forums, etc. (see, e.g., [Agarwal, 2014] for label ex- } \\
\text { amples). }\end{array}$ & \\
\hline Structure & $\begin{array}{l}\text { Frequent machine-generated subject templates, e.g., Your } \\
\text { package number 123 } \rightarrow \text { Your package number * } \\
\text { (see, e.g., Ailon et al. [Ailon et al., 2013] for more details on } \\
\text { subject template generation). }\end{array}$ & \\
\hline Content & $\begin{array}{l}\text { Set of frequent n-grams appearing in the email subject, } \\
\text { e.g., Longest frequent n-gram appearing in the } \\
\text { ['friday lunch', 'lunch invitation'] }\end{array}$ & $\begin{array}{l}\text { Luery, e.g., bob weekly schedule } \rightarrow \\
\text { [ 'weekly schedule'] }\end{array}$ \\
\hline
\end{tabular}

Table 1: Summary of the query and document attribute types. Only attribute values that appear across more than $u$ users in our dataset are considered to be frequent. The infrequent attribute values are discarded.

clicks [Agichtein et al., 2006]). Similarly, if we observe that a document $d$ is often clicked across searches, we may use it as a static a-priori feature of the overall document quality [Richardson et al., 2006].

The case in personal search is different. Users usually do not share documents (e.g., emails or personal files), and therefore directly aggregating click history across users becomes infeasible. To address this problem, instead of directly learning from user behavior for a given $(q, d)$ pair, we instead choose to represent documents and queries using semantically coherent attributes that are in some way indicative of their content.

This approach is schematically described in Figure 3. Both documents and queries are projected into an aggregated attribute space, and the matching is done through that intermediate representation, rather than directly. Since we assume that the attributes are semantically meaningful, we expect that similar personal documents and queries will share many of the same aggregate attributes, making the attribute level matches a useful feature in a learning to rank model.

Some examples of privacy-preserving query-document associations that could potentially be learned by aggregating across a large number of private user interactions are presented in Table 1.

In [Bendersky et al., 2017] we demonstrate that even very highly-dimensional attributes like $\mathrm{n}$-grams can be efficiently incorporated into the learning to rank paradigm described in Section 2.1 without dramatically increasing the size of the feature vector $\mathbf{x}_{q, d}$. This is achieved via an attribute parameterization technique, in which sparse attributes are parameterized using their respective clickthrough rates. [Bendersky et al., 2017] show that for $m$ document attribute types and $n$ query attribute types, attribute parameterization will generate an $m$-dimensional feature vector $\mathcal{P}_{d}$ of query independent features and an $m n$-dimensional feature vector $\mathcal{P}_{q, d}$ of querydependent features.

In general, we will assume that there exists a base score $s c_{b}\left(\mathbf{x}_{q, d}\right)$ for every query-document pair. It can be based on keyword matching or some other ranking features used in private corpora (see, e.g., [Carmel et al., 2015] for an overview). In [Bendersky et al., 2017], we use an adaptive approach, and train the adjustment $\delta\left(\mathcal{P}_{d}, \mathcal{P}_{q, d}\right)$ over the base score $s c_{b}\left(\mathbf{x}_{q, d}\right)$. The scoring function thus becomes $s c_{b}\left(\mathbf{x}_{q, d}\right)+\delta\left(\mathcal{P}_{d}, \mathcal{P}_{q, d}\right)$, which is convenient for our production-environment system, where the base score is already highly optimized, and is disjoint with the newly introduced attribute parameterization features.

The additive nature in this adaptive formulation naturally fits the Multiple Additive Regression Trees (MART) learning algorithm [Hastie et al., 2001]. In every iteration, MART trains a new tree to be added to the existing list of trees. In our setting, we start with the base score $s c_{b}\left(\mathbf{x}_{q, d}\right)$ and then train additive trees over this score.

\subsection{Learning with Biased Feedback}

The position bias model assumes that the observed click modeled by Bernoulli variable $C$ - depends on two factors: (a) whether a user examines a document at position $k$, and (b) whether document $d$ is relevant to query $q$. We can then model the probability of a click as

$$
P(C=1 \mid q, d, k)=\theta_{k} \gamma_{q, d},
$$

where $\theta_{k}$ is the probability that position $k$ is examined, and $\gamma_{q, d}$ is the probability that document $d$ is relevant to query $q$. Note that the model assumes that the examination only depends on the position and the relevance only depends on the query and document, a common assumption in click models [Chuklin et al., 2015].

Both $\theta_{k}$ and $\gamma_{q, d}$ are hidden, and there are several ways to estimate these parameters. It is easy to show that by randomizing the results shown to the user, the expected relevance at each position is constant, and $\theta_{k}$ will be proportional to the number of clicks at position $k$ in the randomized data. However, this can hurt the performance by up to $30 \%$ in live traffic systems [Wang et al., 2018]. To avoid this quality degradation, we can instead resort to random pair inversion, which can significantly reduce the quality decrease. Can we do even better and estimate the position bias directly from click data?

To this end, we propose a novel regression-based EM algorithm [Wang et al., 2018]. Note that in a standard EM algorithm, we would require multiple observations from each query-document pair $(q, d)$ to reliably estimate the relevance $\gamma_{q, d}$. This is not feasible in a personal search scenario where click data is highly sparse. Therefore, in the regression-based EM algorithm we use the feature vector $\mathbf{x}_{q, d}$, and use a function $f$ to compute the relevance: $\gamma_{q, d}=f\left(\mathbf{x}_{q, d}\right)$. The Maximization step attempts to find a regression function $f\left(\mathbf{x}_{q, d}\right)$ to maximize the likelihood given the estimation from the Ex- 


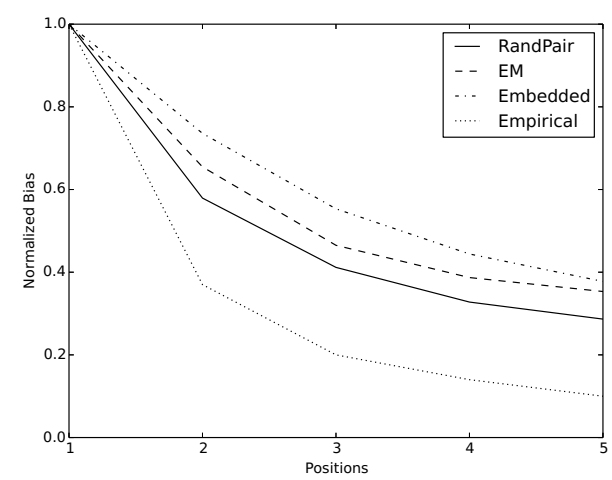

Figure 4: Position bias estimated by several methods and normalized by the top position.

\begin{tabular}{|c|c|}
\hline \multicolumn{2}{|c|}{$\%$ MRR } \\
\hline RandPair Correction & EM Correction \\
\hline+2.14 & +1.94 \\
\hline
\end{tabular}

Table 2: Effects of bias correction on ranking performance. All the differences are reported compared to the unweighted baseline, and are statistically significant.

pectation step. For a detailed description of this process see Algorithm 1 in [Wang et al., 2018].

\section{Experimental Results}

\subsection{Position Bias Estimation}

To evaluate the EM algorithm described in Section 2.3 we examine how well it approximates the empirical position bias that can be obtained using full result randomization. The results are presented in Figure 4, where we compare four alternatives (a) Full result randomization (Empirical), (b) random pair inversion (RandPair), (c) regression-based EM algorithm described in Section $2.3(E M)$, and (d) an embedded approach, where position is directly embedded into the function $g\left(\mathbf{x}_{q, d}, k\right)$ as a feature to approximate bias (Embedded).

As we can see from Figure 4, all the techniques underestimate the Empirical position bias. EM clearly outperforms the Embedded approach, especially at the lower ranks. It achieves an estimation comparable to RandPair, without incurring any loss in quality (as RandPair requires result randomization on live search traffic).

\subsection{Ranking with Biased Feedback}

As shown in Figure 4, clicks are biased. Therefore, when evaluating quality changes using click data in lieu of explicit human ratings (as we do in this paper due to the personal nature of the data), this bias needs to be corrected by incorporating it into the evaluation metric. To this end, [Wang et al., 2016] propose a weighted variant of a standard MRR (mean reciprocal rank) metric. In this variant, query $i$ is weighted by $w_{i}=\frac{1}{b_{k(i)}}$, where $k(i)$ is the click position for query $i$, and $b_{k(i)}$ is the empricial bias at $k(i)$-th position, as shown

\begin{tabular}{|c|c|c|}
\hline & \multicolumn{2}{|c|}{$\%$ MRR } \\
\hline Attribute Type & Query-Independent & Query-Dependent \\
\hline Categories & $+0.48^{*}$ & $+0.80^{* *}$ \\
Structure & $+1.56^{* *}$ & $+1.22^{* *}$ \\
Content & $+1.27^{* *}$ & $+2.11^{* *}$ \\
All & $+2.10^{* *}$ & $+2.60^{* *}$ \\
\hline Full Model & \multicolumn{2}{|c|}{$+\mathbf{3 . 2 4}^{* *}$} \\
\hline
\end{tabular}

Table 3: Overall comparison of different variants. * and ** mean the improvement is significant at 0.05 and 0.01 levels respectively.

in Figure 4. Then, the MRR for a set of queries is defined as $M R R=\frac{1}{\sum_{i} w_{i}} \sum_{i} \frac{w_{i}}{k_{i}}$.

Table 2 shows the effects of correcting the bias when ranking with biased feedback. In both cases, the original training data is reweighted to correct the bias as estimated by either RandPair or EM techniques. As we can see, although the $E M$ algorithm does not require any prior randomization it achieves ranking performance that is roughly $2 \%$ better than the unweighted variant, and statistically indistiguishable from the RandPair algorithm (which requires randomization).

\subsection{Attribute Parameterization Evaluation}

Using the weighted MRR metric presented in the previous section, in Table 3 we evaluate the variants of the attribute parameterization approach described in Section 2.2. In this table, we compare both query-dependent and queryindependent features. For each of them, we train our ranking function by adding each attribute type individually as a feature (the first 3 rows in the table). We then combine all the query-dependent and query-independent parameterized attribute types respectively to form the "all" in the two columns of the table. The "Full Model" uses both the query-dependent and query-independent parameterized attribute types as features in a single ranking function.

From Table 3 we can observe that a combination of all the attribute types outperforms each individual attribute type, resulting in overall improvements of $+2.10 \%$ for queryindependent and $+2.60 \%$ for query-dependent features. This highlights the fact that the selected attribute types are indeed complimentary to each other, and can provide incremental improvements. Further combining all the features and attribute types in the full model results in the best performance, and outperforms the baseline by $+3.24 \%$. These improvements unequivocally demonstrate the importance of crossuser feedback aggregation for personal search quality.

\section{Conclusions}

In this paper we have discussed two novel approaches of dealing with sparsity and bias of user feedback in the personal search setting: query and document attribute parameterization and a regression-based EM algorithm to learn click bias. The proposed approaches are both motivated theoretically as well as demonstrate significant quality improvements in a setting of a large-scale email search engine. They also open up several interesting possibilities for future exploration of other types of bias (e.g., presentation bias [Yue et al., 2010]) and other attribute types. 


\section{References}

[Agarwal, 2014] Shalini Agarwal. Official Gmail Blog: A bit about Bundles in Inbox. https:/gmail.googleblog.com/2014/11/a-bit-aboutbundles-in-inbox.html, 2014.

[Agichtein et al., 2006] Eugene Agichtein, Eric Brill, and Susan Dumais. Improving web search ranking by incorporating user behavior information. In Proceedings of SIGIR, pages 19-26, 2006.

[Ailon et al., 2013] Nir Ailon, Zohar S Karnin, Edo Liberty, and Yoelle Maarek. Threading machine generated email. In Proceedings of WSDM, pages 405-414, 2013.

[Anguera et al., 2008] Xavier Anguera, JieJun Xu, and Nuria Oliver. Multimodal photo annotation and retrieval on a mobile phone. In Proceedings of the 1st ACM International Conference on Multimedia Information Retrieval (MIR), pages 188-194, 2008.

[Bendersky et al., 2017] Michael Bendersky, Xuanhui Wang, Donald Metzler, and Marc Najork. Learning from user interactions in personal search via attribute parameterization. In Proceedings of WSDM, pages 791-799, 2017.

[Carmel et al., 2015] David Carmel, Guy Halawi, Liane Lewin-Eytan, Yoelle Maarek, and Ariel Raviv. Rank by time or by relevance?: Revisiting email search. In Proceedings of CIKM, pages 283-292, 2015.

[Chuklin et al., 2015] Aleksandr Chuklin, Ilya Markov, and Maarten de Rijke. Click Models for Web Search. Morgan \& Claypool, 2015.

[Dumais et al., 2003] Susan Dumais, Edward Cutrell, JJ Cadiz, Gavin Jancke, Raman Sarin, and Daniel C. Robbins. Stuff I've seen: A system for personal information retrieval and re-use. In Proceedings of SIGIR, pages 72-79, 2003.

[Guo and Agichtein, 2012] Qi Guo and Eugene Agichtein. Beyond dwell time: estimating document relevance from cursor movements and other post-click searcher behavior. In Proceedings of WWW, pages 569-578, 2012.

[Guo et al., 2013] Qi Guo, Haojian Jin, Dmitry Lagun, Shuai Yuan, and Eugene Agichtein. Mining touch interaction data on mobile devices to predict web search result relevance. In Proceedings of SIGIR, pages 153-162, 2013.

[Guy et al., 2018] Ido Guy, Alexander Nus, Dan Pelleg, and Idan Szpektor. Care to share?: Learning to rank personal photos for public sharing. In Proceedings of WSDM, pages 207-215, 2018.

[Hastie et al., 2001] Trevor Hastie, Robert Tibshirani, and Jerome Friedman. The Elements of Statistical Learning. Springer, 2001.

[Joachims et al., 2005] Thorsten Joachims, Laura Granka, Bing Pan, Helene Hembrooke, and Geri Gay. Accurately interpreting clickthrough data as implicit feedback. In Proceedings of SIGIR, pages 154-161, 2005.
[Joachims, 2002] Thorsten Joachims. Optimizing search engines using clickthrough data. In Proceedings of KDD, pages 133-142, 2002.

[Kamvar et al., 2009] Maryam Kamvar, Melanie Kellar, Rajan Patel, and Ya Xu. Computers and iPhones and mobile phones, oh my!: A logs-based comparison of search users on different devices. In Proceedings of WWW, pages 801$810,2009$.

[Liu, 2009] Tie-Yan Liu. Learning to rank for information retrieval. Foundations and Trends in Information Retrieval, 3(3):225-331, 2009.

[Richardson et al., 2006] Matthew Richardson, Amit Prakash, and Eric Brill. Beyond PageRank: machine learning for static ranking. In Proceedings of $W W W$, pages 707-715, 2006.

[Richardson et al., 2007] Matthew Richardson, Ewa Dominowska, and Robert Ragno. Predicting clicks: Estimating the click-through rate for new ads. In Proceedings of $W W W$, pages 521-530, 2007.

[Tromba et al., 2017] Isabella Tromba, John Gallagher, and Jason Liszka. Search at Slack. https://slack.engineering/search-at-slack-431f8c80619e, 2017.

[Wang et al., 2016] Xuanhui Wang, Michael Bendersky, Donald Metzler, and Marc Najork. Learning to rank with selection bias in personal search. In Proceedings of SIGIR, pages 115-124, 2016.

[Wang et al., 2018] Xuanhui Wang, Nadav Golbandi, Michael Bendersky, Donald Metzler, and Marc Najork. Position bias estimation for unbiased learning to rank in personal search. In Proceedings of WSDM, pages 610-618, 2018.

[Yue et al., 2010] Yisong Yue, Rajan Patel, and Hein Roehrig. Beyond position bias: Examining result attractiveness as a source of presentation bias in clickthrough data. In Proceedings of $W W W, 2010$. 